\section{PL-2 Graphene future emerging technology}

\author{
Andrea Ferrari ${ }^{1}$
}

1. Cambridge Graphene Centre Engineering Department, University of Cambridge, UK

email: acf26@eng.cam.ac.uk

Disruptive technologies are usually characterised by universal, versatile applications, which change many aspects of our life simultaneously, penetrating every corner of our existence. In order to become disruptive, a new technology needs to offer not incremental, but dramatic, orders of magnitude improvements. Moreover, the more universal the technology, the better chances it has for broad base success. Does graphene have a chance to become the next disruptive technology? Can graphene be the material of the 21 th century? Are the properties of graphene so unique to overshadow the unavoidable inconveniences of switching to a new technology, a process usually accompanied by large $R \& D$ and capital investments? In spite of the inherent novelty associated with graphene and the lack of maturity of graphene technology, a roadmap can be envisaged, including short-term milestones, and some medium- to long-term targets, intrinsically less detailed, but potentially even more disruptive. This should guide the transition towards a technological platform underpinned by graphene, with opportunities in many fields and benefits to society.

Keywords: graphene 